\title{
Influence of ABCB1 C3435T and ABCG2 C421A Gene Polymorphisms in Response to Imatinib Mesylate in Chronic Myeloid Leukemia Patients
}

\author{
A. Anthony, R. Ankathil, G. Ai-Sim, S. A. W. Fadilah, and A. A. Baba
}

\begin{abstract}
Despite the excellent efficacy results of IM treatment in CML patients, resistance to IM has emerged as a significant problem. Genetic variations in genes involved in drug transportation encoding membrane transporters such as ABCB1 and ABCG2 might influence the pharmacokinetic and metabolism of IM. The genotype of a patient is increasingly recognized in influencing the response to the treatment. In this study, the relationship between IM response and 2 SNPs (ABCB1 C3435T and ABCG2 C421A) were investigated among Malaysian CML patients undergoing IM treatment. Peripheral blood was withdrawn from 91 healthy individuals and 45 CML patients undergoing IM treatment. DNA was extracted from the blood samples and followed by the genotyping technique using PCR-RFLP method. The results showed the homozygous wild type genotype (CC) of SNP ABCB1 C3435T was higher in CML patients showing IM resistant compared to IM good response CML patients with $\boldsymbol{p}=\mathbf{0 . 0 0 4}$. Interestingly, IM resistance was lower for patients homozygous for the A allele of ABCG2 SNP C421A compared to patients with $C$ allele ( $33 \%$ vs. $64 \%$, $p=0.0001$ ). Besides, these 2 SNPs were found not to contribute to the genetic susceptibility of CML when evaluated with healthy control subjects. These preliminary results prompt us to suggest the possibility of exploring these SNPs as biomarkers to predict the response to IM in CML patients prior to IM treatment.
\end{abstract}

Index Terms-ABCB1 C3435T, ABCG2 C421A, polymorphisms, imatinib mesylate, chronic myeloid leukemia (CML)

\section{INTRODUCTION}

Chronic Myeloid Leukemia (CML), a myeloproliferative disorder, comprises $14 \%$ of all leukemias. The synthetic tyrosine kinase inhibitor Imatinib mesylate (IM), also known as Glivec or Gleevec, has been well documented as first line treatment for CML. Despite the excellent results encountered with IM therapy, many CML patients in advanced phases do not respond to IM, others who initially respond to IM may eventually develop resistant disease.

Development of resistance to IM is a multifactorial phenomenon in patients with CML and may be mediated by

Manuscript received February 1, 2012; revised March 1, 2012. This work was supported in part by the Universiti Sains Malaysia under Research University Grant 1001/PPSP/812067.

A. Au and R. Ankathil are with the Human Genome Centre, School of Medical Sciences, Universiti Sains Malaysia, Malaysia. (email:auzl@live.com; rankathil@hotmail.com)

A. S. Goh is with Hospital Pulau Pinang, Malaysia.

F. S. A. Wahid is with the Cell Therapy Centre, UKM Medical Centre, Universiti Kebangsaan Malaysia, Malaysia.

A. A. Baba is the Dean, School of Medical Sciences, Universiti Sains Malaysia, Malaysia (email: ababa@kb.usm.my) diverse mechanisms. However, there are 2 broad mechanisms of resistance. (1) BCR-ABL dependent pathways and (2) BCR-ABL independent pathways [1]. BCR-ABL dependent pathways include BCR-ABL Tyrosine Kinase domain mutations and BCR-ABL gene amplifications. But several CML patients showing IM resistance were identified who do not fit into the BCR-ABL dependent mechanisms. It is presumed that the mechanisms of IM resistance in such patients might be mediated through BCR-ABL independent pathways. BCR-ABL independent pathways of IM resistance may include several mechanisms. Not much data are available on this issue as very few studies have been carried out on this aspect worldwide and nil from Malaysia.

Membranebound efflux transporters play important roles in mediating chemosensitivity and resistance of tumour cells. There are seven subfamilies (ABCA through to ABCG). Members of the ABC family are important in the mechanism of multidrug resistance. Important genes encoding $A B C$ transporters associated with multidrug resistance (MDR1) include ABCB1 encoding P-Glycoprotein (PGP), also known as ABCB1 with protein product MRP1 and ABCG2 also named MXR (mitoxanthrone resistance protein) or BCRP (Breast Cancer Resistance Protein).

Of these transporters, ABCB1 and ABCG2 are involved in IM transport. P-glycoprotein, a member of the adenosine triphosphate (ATP) binding casette family of membrane transporters, is well known to be expressed on the surface of cancer cells and to be associated with multidrug resistance of tumour cells and affect the pharmacokinetics of many drugs and xenobiotics [2]. It can potentially play a part in resistance to IM, either by transporting the drug out from the target cell or from gastrointestinal tract. ABCG2 is another transporter protein mediating drug resistance. The expression of ABCG2 has not been well worked out in cancer. Both ABCB1 and ABCG2 are expressed at the apical membrane of the small intestine and bile canalicular membranes, and gastrointestinal transport activity could orally affect IM bioavailability. In humans, this multidrug transporter plays a key role in determining drug bioavailability and difference in drug response has been reported to exist amongst different ethnic groups [3], [4], [5].

With the recent advances in pharmacogenomics, genetic polymorphisms have been revealed as one of the most important factors influencing the pharmacokinetics and pharmacodynamics of many drugs. Polymorphisms or nucleotide diversity occurring in the genes encoding drug metabolizing enzymes, transporters and target molecules may affect the pharmacokinetic and pharmacodynamics of 
respective drugs. Single nucleotide polymorphisms (SNPs) in ABCB1 and ABCG2 genes have the potential to alter protein function and could also influence the efficacy of absorption or elimination [6].

Due to the importance of determining the role of SNPs in the response to the CML treatment, this study was designed to investigate the allele and genotype frequencies of SNPs ABCB1 C3435T and ABCG2 C421A in CML patients undergoing IM treatment and to determine whether different genotype pattern of these SNPs have any influence in mediating good response and resistance to IM. The second objective was to source out the allele \& genotype frequencies between normal controls \& CML patients in our population and evaluate their contributory role in mediating CML susceptibility risk.

\section{MAteriAls AND METHODS}

\section{A. Sample Collection}

In this multi-centric study, Philadelphia (Ph) chromosome positive CML patients undergoing IM treatment in various centres in Malaysia were recruited. $\mathrm{Ph}$ positive CML patients (Chronic phase or Accelerated phase), treated with standard dose IM (400mg) on frontline treatment and who demonstrated the following clinical features were classified as IM resistant CML patients [7]:

- No major or complete cytogenetic response by 12 months after initiation of therapy, loss of complete cytogenetic response or development of IM resistance

- Failure to achieve a complete hematologic response after 3 months

- No major molecular response for at least 6 months

Those CML patients showing very good response to IM, at the cytogenetic and molecular level (good responders) were also included for comparison of results. After obtaining written-informed consent, $3 \mathrm{ml}$ peripheral blood was collected from 45 CML patients and 91 normal healthy individuals in EDTA vacutainer tubes. DNA from the peripheral blood of the study subjects were extracted using QIAGEN amp DNA extraction kit (Qiagen, Hilden, Germany) following the manufacturer's protocol. Genotyping

\section{B. Genotyping}

The polymerase chain reaction-restriction fragment length polymorphism (PCR-RFLP) method was used for the genotyping of $3435 \mathrm{C}>\mathrm{T}$ and C421A SNPs. For the 3435 $\mathrm{C}>\mathrm{T}$ SNP, the sequences of oligonucleotide primers used were forward primer 5'-TGCTGGTCCT GAAGTTGATC TGTGAAC-3' and reverse primer 5'-ACATTAGGCA GTGACTCGAT GAAGGCA-3' [8]. The reagents and conditions for PCR amplification consisted of $10 \mathrm{X}$ PCR buffer, $200 \mu \mathrm{mol}$ deoxynucleoside-5'-triphosphate (dNTPs), $0.2 \mu \mathrm{mol}$ of each specific primer, $1.5 \mathrm{mmol} \mathrm{MgCl}_{2}, 1$ unit AmpliTaq Gold DNA Polymerase and 50 ng genomic DNA templates in a $25 \mu \mathrm{l}$ reaction. PCR conditions were $95^{\circ} \mathrm{C}$ for 3 minutes, followed by 35 cycles of $95^{\circ} \mathrm{C}$ for 30 seconds, $61^{\circ} \mathrm{C}$ for 30 seconds and $72^{\circ} \mathrm{C}$ for 30 seconds with a final 5 minutes cycle at $72^{\circ} \mathrm{C}$. The PCR products were digested with restriction enzyme Fermentas FastDigest Mbo I and DNA fragments generated after restriction enzyme digestion were separated on 3\% of Bioline Agarose HiRes gel (Bioline, London, UK) with $0.1 \%$ of GelRed DNA stain. For the genotyping of $421 \mathrm{C}>\mathrm{A}$ SNP, the primer sequences used were forward primer 5'-GTTGTGATGGGCACTCTGATGGT-3' and reverse primer 5'-CAAGCCACTTTTCTCATTGTT-3' [9]. The reaction mixture for PCR amplification consisted of $10 \mathrm{X}$ PCR buffer, $200 \mu \mathrm{mol}$ dNTPs, $0.2 \mu \mathrm{mol}$ of each specific primer, $1.5 \mathrm{mM} \mathrm{MgCl}_{2}$, 1 unit AmpliTaq Gold DNA Polymerase and $50 \mathrm{ng}$ genomic DNA templates and PCR grade water was added to a final volume of $25 \mu \mathrm{L}$. PCR amplification consisted of 3 steps: denaturation at $95^{\circ} \mathrm{C}$ for 30 seconds, annealing at $60^{\circ} \mathrm{C}$ for 30 seconds, and extension at $72^{\circ} \mathrm{C}$ for 30 seconds after denaturation for $95^{\circ} \mathrm{C}$ for 3 minutes, followed by 35 cycles. PCR products digested with Fermentas FastDigest restriction enzyme Taa I and the DNA fragments generated were run on $5 \%$ of Bioline Agarose HiRes gel (Bioline, London, UK) with $0.1 \%$ of GelRed DNA stain. Allele and genotype frequencies for each SNP in all normal subjects were determined.

\section{Statistical Analysis}

The difference in allele and genotype of ABCB1 C3435T and ABCG2 C421A frequencies among patients undergoing imatinib treatment and normal controls were determined by using the chi-square test or Fisher's exact test (if $n<5$ ). StatCalc- EpiInfo version 6 was used to determine the Odds ratio for evaluating the risk association of ABCB1 C3435T and ABCG2 C421A SNPs on CML susceptibility risk. All tests were two-sided, and the $P$ value when $<0.05$ was considered as significant.

\section{RESUlTS AND DisCUSSION}

We analyzed samples obtained from 91 healthy subjects and $45 \mathrm{CML}$ patients to detect ABCB1 and ABCG2 polymorphisms in positions of 3435 and 421 respectively. Within the 45 CML subjects included, $48.9 \%$ (22) were IM responders and $51.1 \%$ (23) IM non-responders to the standard dose of IM (400 mg/day) after classified accordingly to [7]. Figure 1 was the genotype results for ABCB1 C3435T polymorphisms. The homozygous wild type CC, heterozygous CT and homozygous variant TT was classified into different band sizes after digest by the specific restriction enzyme (Mbo I). Figure 2 also the result in the different band sizes in order to categorize the variant of genotypes pattern after digest with Taa I restriction enzyme.

The introduction of IM had a marked impact on treatment outcomes in CML patients. Despite the excellent response obtained, resistance to IM has emerged as a daunting problem in the management of CML patients. The molecular basis for inter-individual variation in IM response is still unclear. While a number of factors may contribute to inter-individual variability, the genotype of a patient is increasingly implicated in influencing drug disposition and activity. SNPs in the human genome contribute to wide variations in how individuals response to medications either by changing the 
pharmacokinetics of drugs or by altering the cellular response to therapeutic agents [10]. Both ABCB1 (MDR1) and ABCG2 (BCRP) have been demonstrated to display high affinity for IM and confer IM resistance in vitro by extruding IM from hematopoietic cells [11], [12]. Moreover, both transporters have shown elevated expression profile in CML stem cells [13]. Several known SNPs have been reported to modify their activity [14], [15]. The silent C3435T polymorphism (rs1045642) in exon 26 of $A B C B 1$ was demonstrated to be a functional polymorphism altering mRNA stability, modifying the P-gp expression and consequently reducing its' drugs substrate specificity. The SNP C421A (rs2231142) which substitutes a glutamine for a lysine, is the most frequent non-synonymous polymorphism in $A B C G 2$. We undertook this study to investigate whether the SNPs C3435T and C421A could play any contributory role in mediating response to IM in Malaysian CML patients.

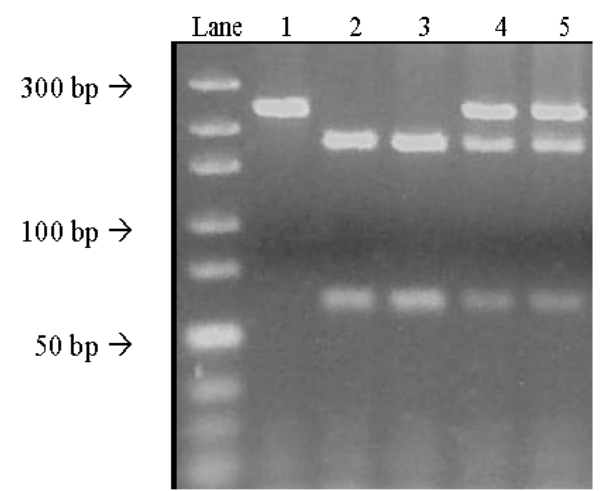

Fig. 1. Electrophoresis patterns for MDR1 alleles analyzed by PCR-RFLP. L: O'GeneRuler Ultra Low Range DNA Ladder, 10-300 bp ; lane 1: homozygous T allele (248bp); lane 2 \& 3: homozygous C allele (172bp, 60bp, 16bp); lane 4 \& 5: heterozygous (248bp, 172bp, 60bp, 16bp)

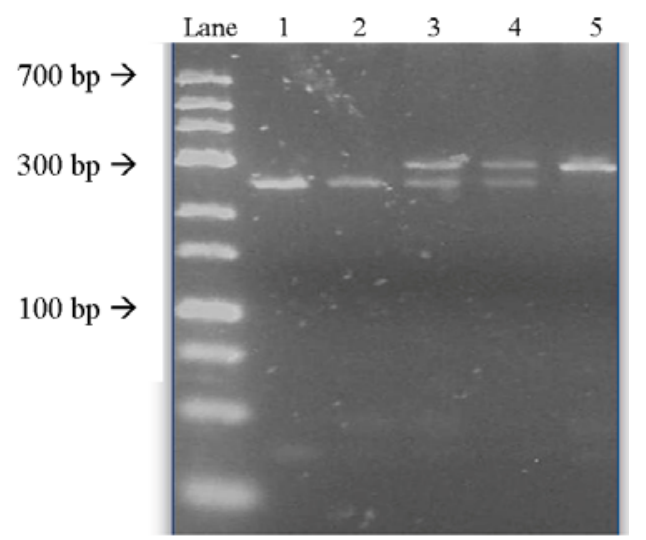

Fig. 2. Electrophoresis patterns for MDR1 alleles analyzed by PCR-RFLP. L: O'GeneRuler Low Range DNA Ladder, 25-700 bp; lane 1 \& 2: homozygous C allele (251bp, 21bp, 17bp); lane 3 \&4: heterozygous (268bp, 251bp, 21bp, 17bp); lane 5: homozygous A allele (268bp, 21bp)

In our study, the overall frequency of the ABCB1 3435 CC, CT, and TT genotypes was 36.3, 49.4, and 14.3\%, respectively in healthy control group and 35.6, 48.8 and $15.6 \%$ among CML patients as shown in Table I. There was no significant difference in the genotype and allele frequencies of ABCB1 C3435T between the CML patients and healthy subject group, suggesting a lack of relationship between these variants and the risk for CML $(p>0.05)$. However with regard to ABCG2 C421A polymorphism, the frequency of heterozygous (CA) $55.6 \%$ and homozygous variant genotypes (AA) 20\% were higher in CML patients compared to frequencies of heterozygous (45\%) and homozygous variant (13.2\%) among healthy controls, but statistically insignificant ( $p=0.278,0.321$ respectively). Comparison of the allele frequencies of $\mathrm{C}$ and A of ABCG2 C421A between CML patients and controls showed the variant allele A to be higher in CML patients but statistically insignificant ( $p=0.115$ ). From the data observed, it seems that the genotype frequencies of homozygous wild type, heterozygous and homozygous variant of SNPs ABCB1 C3435T and ABCG2 C421A between CML patients and controls were not significantly different. The association of variant genotypes of these 2 SNPs with CML susceptibility was evaluated and the Odds ratio for ABCB1 C3435T and ABCG2 C421A polymorphisms determined are shown in Table II . The data indicated no association of these variant genotypes with the susceptibility risk of CML.

TABLE I: FREQUENCY OF ABCB1 C3435T AND ABCG2 C421A POLYMORPHISMS IN HEALTHY CONTROLS AND CML PATIENTS

\begin{tabular}{lcccc}
\hline \multirow{2}{*}{$\begin{array}{l}\text { Gene } \\
\text { Polymorphism }\end{array}$} & $\begin{array}{c}\text { Genotype } \\
\text { / Allele }\end{array}$ & $\begin{array}{c}\text { Controls } \\
\mathrm{n}=91\end{array}$ & $\begin{array}{c}\text { CML } \\
\mathrm{n}=45\end{array}$ & $p$-value \\
& & $33(36.3)$ & $16(35.6)$ & 0.999 \\
ABCB1 & $\mathrm{CC}$ & $45(49.4)$ & $22(48.8)$ & 0.720 \\
C3435T & $\mathrm{CT}$ & $13(14.3)$ & $7(15.6)$ & 0.8037 \\
& $\mathrm{TT}$ & 0.61 & 0.60 & 0.999 \\
& $\mathrm{C}$ & 0.39 & 0.40 & \\
& $\mathrm{~T}$ & & & \\
ABCG2 & & $38(41.8)$ & $11(24.4)$ & 0.058 \\
C421A & $\mathrm{CC}$ & $41(45.0)$ & $25(55.6)$ & 0.278 \\
& $\mathrm{CA}$ & $12(13.2)$ & $9(20)$ & 0.321 \\
& AA & 0.64 & 0.52 & 0.115 \\
& C & 0.36 & 0.48 & \\
\hline
\end{tabular}

TABLE II: ODDS RATIO OF ABCB1 C3435T AND ABCG2 C421A POLYMORPHISMS IN HEALTHY CONTROLS AND CML PATIENTS

\begin{tabular}{|c|c|c|c|c|c|}
\hline \multirow{2}{*}{$\begin{array}{l}\text { Gene } \\
\text { Polymorphism }\end{array}$} & \multirow{2}{*}{$\begin{array}{l}\text { Genotype } \\
\text { / Allele }\end{array}$} & \multicolumn{3}{|c|}{0.95 Confidence } & \\
\hline & & $\begin{array}{l}\text { Odds } \\
\text { Ratio }\end{array}$ & $\begin{array}{l}\text { Lower } \\
\text { Limit }\end{array}$ & $\begin{array}{l}\text { Upper } \\
\text { Limit }\end{array}$ & $\begin{array}{c}p \text {-valu } \\
\mathrm{e}\end{array}$ \\
\hline & $\mathrm{CC}$ & & & & \\
\hline$A B C B 1$ & CT & 1.01 & 0.43 & 2.38 & 0.983 \\
\hline C3435T & $\mathrm{TT}$ & 1.11 & 0.32 & 3.78 & 0.851 \\
\hline $\begin{array}{l}A B C G 2 \\
C 421 A\end{array}$ & $\begin{array}{l}\mathrm{CC} \\
\mathrm{CA} \\
\mathrm{AA}\end{array}$ & $\begin{array}{l}2.81 \\
2.59\end{array}$ & $\begin{array}{l}0.85 \\
0.76\end{array}$ & $\begin{array}{l}5.30 \\
8.93\end{array}$ & $\begin{array}{l}0.078 \\
0.083\end{array}$ \\
\hline
\end{tabular}

Table III shows the ABCB1 C3435T and ABCG2 C421A genotype and allele frequencies between good responder and non-responder CML patients. For ABCB1 C3435T, the frequency of homozygous variant TT was higher in IM resistant CML patients (17.4\%) compared to IM good response CML patients (13.6\%), but the different was not statistically significant ( $p=0.999)$. For ABCG C421A, the heterozygous $(\mathrm{CA}=63.6 \%)$ and homozygous wild type $(\mathrm{CC}=$ $31.9 \%)$ genotype frequencies were comparatively higher in CML patients showing IM good response compared to IM resistant CML patients but statistically insignificant ( $p=0.373,0.071$ respectively). 
Recently Duluq et al [16] has reported that ABCB/MDR1 polymorphisms are associated with major molecular responses to standard dose IM and the SNP $3435 \mathrm{C}>\mathrm{T}$ has been repeatedly shown to predict changes in the function of P-Gp. Study by Deenik and colleagues [17] also reported that patients with homozygous variant T3435T showed lower probabilities to obtain a Major Molecular Response (MMR) and Complete Molecular Response. Similarly, higher resistance rate in homozygous variant $A B C B 1$ T3435T allele was reported by Ni et al [18]. Even though statistically insignificant our results are also in favour of these findings. The small sample size in our study might have hindered in deriving a significant association with adequate statistical power. Another study conducted by Kim et al [19] on CML patients, did not find an association between $A B C B 1$ polymorphisms and MMR. Frequencies with which these polymorphisms exist in a population have also been shown to be ethnically related [20]. This could be a reason for the inconsistency in results.

TABLE III: FREQUENCY OF ABCB1 C3435T AND ABCG2 C421A POLYMORPHISMS IN CML PATIENTS WITH GOOD RESPONSE OR RESISTANCE TO IM TREATMENT

\begin{tabular}{lcccl}
\hline $\begin{array}{l}\text { Gene } \\
\text { Polymorphism }\end{array}$ & $\begin{array}{l}\text { Genotype } \\
\text { / Allele }\end{array}$ & $\begin{array}{c}n \text { IM frequency (\%) } \\
\text { IM } \\
\text { responsive } \\
\mathrm{n}=22\end{array}$ & $\begin{array}{c}\text { IM } \\
\text { resistance } \\
\mathrm{n}=23\end{array}$ & $\begin{array}{l}\text { p-valu } \\
\text { e }\end{array}$ \\
\hline \multirow{3}{*}{ ABCB1 } & $\mathrm{CC}$ & $6(27.3)$ & $10(43.5)$ & 0.353 \\
C3435T & $\mathrm{CT}$ & $13(59.1)$ & $9(39.1)$ & 0.366 \\
& $\mathrm{TT}$ & $3(13.6)$ & $4(17.4)$ & 0.999 \\
& $\mathrm{C}$ & 0.57 & 0.63 & 0.312 \\
& $\mathrm{~T}$ & 0.43 & 0.37 & \\
& $\mathrm{CC}$ & $1(4.5)$ & $10(43.5)$ & 0.004 \\
ABCG2 & $\mathrm{CA}$ & $14(63.6)$ & $11(47.8)$ & 0.373 \\
C421A & $\mathrm{AA}$ & $7(31.9)$ & $2(8.7)$ & 0.071 \\
& $\mathrm{C}$ & 0.36 & 0.67 & 0.0001 \\
& $\mathrm{~A}$ & 0.64 & 0.33 & \\
\hline
\end{tabular}

Meanwhile for ABCG2 C421A, the homozygous major CC genotype with a frequency of $43.5 \%$, was found significantly to be higher among IM resistant group compared to IM good response CML patients with a frequency of only $4.3 \%$ ( $p=0.004)$. When the frequency of variant allele A was compared between CML patients showing good response and resistance to IM (0.64 vs 0.33 ), the frequency was significantly higher in CML patients with good response $(p=0.0001)$ indicating that BCRP efflux transporter encoded by ABCG2 might have an important role in transporting IM. Our results had shown that carriers of the C421C allele of ABCG2 had more resistance to IM, suggesting that the increase of risk to resistance to IM could be related to the presence of the $\mathrm{C}$ allele. Likewise, ABCG2 C421A genotyping done in Japanese population had shown correlation with respect to MMR and IM trough concentration, in the study by Takahashi et al [21]. Further prospective studies in larger populations and Meta Analysis by combination of several studies are needed to determine the effects of these ABCB1 and ABCG2 gene haplotypes on IM treatment which may provide more power to detect small but clinically significant effects and also to overcome the bias.

\section{CONCLUSION}

The preliminary results of our study demonstrated a significant correlation of the SNP ABCG2 C421A with imatinib efficacy and a lack of significant association of SNP ABCB1 C3435T with IM response in Malaysian CML patients.

\section{ACKNOWLEDGMENT}

A. Au is a recipient of USM Fellowship 01/11. We wish to acknowledge Dr. Chew Teng Keat, Dr. Azlan Husin, Dr. Abu Dzar Abdullah for their help in collecting CML patient samples.

\section{REFERENCES}

[1] A. Hochhaus, "Chronic myelogenous leukemia (CML) resistance to tyrosine kinase inhibitors,” Ann Oncol, vol. 17, pp. 274 - 279, 2006.

[2] J. V. Melo, and C. Chuah, "Resistance to imatinib mesylate in chronic myeloid leukaemia,” Cancer Letters, vol. 249, pp. 121-132, 2007.

[3] I. Cascorbi, Gerloff, and T. A. Johne. Frequency of single nucleotide polymorphisms in the P-glycoprotein drug transporter MDRI gene in white subjects. Clin Pharmacol Ther. vol.69, pp. 69-74, 2001.

[4] K. Tang, S. M. Ngoid, P. C. Gweea, M. Z. John, D. Chuaa, J. D. Edmund, Lee, S. Samuel, C. Chong, G. L. Caroline, and D. Lee, "Distinct haplotype profiles and strong linkage disequilibrium at the MDR1 multidrug transporter gene locus in three ethnic Asian populations,” Pharmacogenetics, vol. 12, pp. 437-450, 2002.

[5] C. Kimchi-Sarfaty, A.H. Marple, and S. Shinar, "Ethnicity related polymorphisms and haplotype in the human ABCB1 gene," Pharmacogenomics, vol. 8, no.1, pp. 29-39, 2007.

[6] H. Gurney, M. Wong, R. L. Balleine, L. P. Rivory, A. J. McLachlan, J. M. Hoskins, N. Wilcken, C. L. Clarke, G. J. Mann, M. Collins, S. E. Delforce, K. Lynch, and H. Schran, "Imatinib disposition and ABCB1 (MDR1, P-glycoprotein) genotype,” Clin Pharmacol Ther., vol. 82, pp. 33-40, 2007.

[7] M. Baccarani, G. Saglio, J. Goldman, A. Hochhaus, B. Simonsson, F. Appelbaum, J. Apperley, F. Cervantes, J. Cortes, M. Deininger, A. Gratwohl, F. Guilhot, M. Horowitz, T. Hughes, H. Kantarjian, R. Larson, D. Niederwieser, R. Silver and R. Hehlmann, "Evolving concepts in the management of chronic myeloid leukemia: recommendations from an expert panel on behalf of the European LeukemiaNet,” Blood, vol. 108, pp.1809-1820, 2006.

[8] M.M. Ameyaw, F. Regateiro, and T. Li, "MDR1 pharmacogenetics: frequency of the C3435T mutation in exon 26 is significantly influenced by ethnicity,” Pharmacogenetics, vol. 11, pp. 217-221, 2001.

[9] D. Kobayashi, I. Ieiri, T. Hirota, H. Takane, S. Maegawa, J. Kigawa, H. Suzuki, E. Nanba, M. Oshimura, N. Terakawa, K. Otsubo, K. Mine, and Y. Sugiyama, "Functional Assessment of ABCG2 (BCRP) Gene Polymorphisms to Protein Expression in Human Placenta,” Drug Metabolism and Disposition, vol. 33, pp. 94-101, 2005.

[10] Z. E. Sauna, C. Kimchi-Sarfaty, S. V. Ambudkar and M. M. Gottesman, "Silent polymorphisms speak: how they affect pharmacogenomics and the treatment of cancer," Cancer Res, vol. 67, 9609-9612.

[11] F. X. Mahon, F. Belloc, V. Lagarde, C. Chollet, F. Moreau-Gaudry, J. Reiffers, J.M. Goldman, and J.V. Melo. MDR1 gene overexpression confers resistance to imatinib mesylate in leukemia cell line models. Blood. 2003, 101: 2368-2373.

[12] T. Nakanishi, K. Shiozawa, B.A. Hassel, and D.D. Ross. Complex interaction of BCRP/ABCG2 and imatinib in BCR-ABL-expressing cells: BCRP-mediated resistance to imatinib is attenuated by imatinib-induced reduction of BCRP expression. Blood. 2006, 108: 678-684.

[13] N. E. Jordanides, H. G. Jorgensen, T. L. Holyoake, and J. C. Mountford. Functional ABCG2 is overexpressed on primary CML CD34+ cells and is inhibited by imatinib mesylate. Blood. 2006, 108: 1370-1373. 
[14] E. R. Gardner, H. Burger, R. H. van Schaik, A. T. van Oosterom, E. A. de Bruijn, G. Guetens, H. Prenen, F.A. de Jong, S.D. Baker, S.E. Bates, W. D. Figg, J. Verweij, A. Sparreboom, and K. Nooter. Association of enzyme and transporter genotypes with the pharmacokinetics of imatinib. Clin. Pharmacol. Ther. 2006, 80: 192-201.

[15] K. Morisaki, R. W. Robey, C. Ozvegy-Laczka, Y. Honjo, O. Polgar, K. Steadman, B. Sarkadi, S.E. Bate. Single nucleotide polymorphisms modify the transporter activity of ABCG2. Cancer Chemother. Pharmacol. 2005, 56: 161-172.

[16] S. Dulucq, S. Bouchet, B. Turcq, E. Lippert, G. Etienne, J. Reiffers, M. Molimard, M. Krajinovic, F.X. Mahon. Multidrug resistance gene (MDR1) polymorphisms are associated with major molecular responses to standard-dose imatinib in chronic myeloid leukemia. Blood. 2008, 112:2024-2027.

[17] W. Deenik, B. Van der Holt and J. Janseen. Polymorphisms in the Multidrug Resistance gene MDR1 (ABCB1) predict for molecular resistance in patients with newly diagnosed chronic myeloid leukemia (CML) receiving high dose imatinib. Blood. 2010, 116: pp. 6144-6145, 2007.
[18] L. N. Ni, J. Y. Li, K. R. Miao, C. Qiao, S. J. Zhang, H. R. Qiu, and S $\mathrm{X}$. Qian, 'Multidrug resistance gene (MDR1) polymorphisms correlate with imatinib response in chronic myeloid leukemia," Med Oncol., vol. 28, pp. 265-269, 2010.

[19] D. H. Kim, L. Sriharsha, W. Xu, S. Kamel-Reid, X. Liu, K. Siminovitch, H.A. Messner and J.H. Lipton, "Clinical relevance of a pharmacogenetic approach using multiple candidate genes to predict response and resistance to imatinib therapy in chronic myeloid leukemia,” Clin Cancer Res, vol. 15, pp. 4750-4758, 2007.

[20] C. Cropp, S. Yee, and K. Giascomini, "Genetic variation in drug transporters in ethnic populations," Clinical Pharmacology \& Therapeutics, vol. 84, pp. 412-416, 2008.

[21] N. Takahashi, M. Miura, S. A. Scott, H. Kagaya, Y. Kameoka, H. Tagawa, H. Saitoh, N. Fujishima, T. Yoshioka, M. Hirokawa and K. Sawada, "Influence of CYP3A5 and drug transporter polymorphisms on imatinib trough concentration and clinical response among patients with chronic phase chronic myeloid leukemia," J Human Genet. vol. 55, pp. 731-737, 2010. 